\title{
Las bases biopsicológicas de la imputabilidad en la conducta impulsiva
}

\author{
The biopsychological bases of criminal responsibility in \\ impulsive behaviour
}

\section{Resumen}

Un repaso bibliográfico sobre la impulsividad nos muestra diferentes concepciones de esta conducta en la que pueden intervenir distintas dimensiones. Vemos como la impulsividad puede ser definida como respuesta a estímulos con carga afectiva y con prevalencia de factores motóricos en ausencia de deliberación. Pero también encontramos que en las conductas impulsivas intervienen elementos cognitivos de deliberación y temperamentales-emocionales como el de "búsqueda de sensaciones" descrito por Zuckerman.

Nosotros, atendiendo a la presencia de los distintos factores de la impulsividad, hemos clasificado estas conductas en impulsivas inmediatas e impulsivas diferidas. Las primeras serían característica, entre otros, del trastorno explosivo intermitente y de los trastornos límite y antisocial de personalidad. Las segundas, aparecerían, por ejemplo, en el juego patológico y piromanía y podrían caracterizar determinados comportamientos de sujetos antisociales.

Las distintas dimensiones de la conducta impulsiva pueden influir sobre las bases biopsicológicas de la imputabilidad tanto en los sujetos con impulsividad inmediata como en los que presentan conductas impulsivas diferidas.

Palabras clave: Conducta impulsiva. Impulsividad. Trastorno en el control de los impulsos.

\section{Abstract}

A review of the available literature on impulsivity reveals different conceptions of this behaviour in which various dimensions may play a role. Impulsivity may be defined as a response to emotionally charged stimuli with a prevalence of kinetic factors in an absence of deliberation. Examples can also be found in which cognitive factors of deliberation and a temperamental-emotional nature intervene in impulsive behaviour such as the "sensations seeking " described by Zuckerman.

Given the presence of these different factors in impulsivity, we have classified the behaviours into immediate impulsive behaviour and deferred impulsive behaviour. The former are characteristic of intermittent explosive disorders and borderline and anti-social personality disorders. The latter appear, for example, in pathological gambling and pyromania and may characterise certain anti-social behaviours.

The different dimensions of impulsive behaviour may have an influence over the bio-psychological bases of criminal responsibility in individuals with immediate impulsivity and in those with deferred impulsive behaviour.

Key words: Impulsivity. Impulsive behaviour. Impulse control disorder.

\section{Introducción}

La impulsividad tiene interés clínico pues es un síntoma común de muchas enfermedades mentales, es propia de determinados trastornos de personalidad o puede constituir el criterio fundamental de diagnóstico de trastornos que cursan con dificultades en el control de los impulsos.

También resulta relevante desde la perspectiva forense, ya que los comportamientos impulsivos son

\section{A. Villarejo Ramos}

Médico forense. Instituto de Medicina Legal de Cádiz. Especialista en Medicina Legal y Forense. Especialista Universitario en Psiquiatría Legal y Forense.

Correspondencia: Dr. Alberto Villarejo. E-mail: alberto.villarejo @juntadeandalucia.es
Fecha de recepción: 1.MAR.2012

Fecha de aceptación: 27.MAY.2012 
el origen de no pocas conductas ilícitas. El mayor reto médico-legal supone, en nuestra opinión, la determinación de la influencia de la impulsividad en los actos volitivos y en el libre albedrío, eje fundamental sobre el que gira la imputabilidad del sujeto infractor.

El estudio de los distintos factores que intervienen en la impulsividad nos ayudará a establecer las eventuales alteraciones psicopatológicas que modifican la responsabilidad penal.

\section{Concepto de impulsividad}

Según los conceptos de los distintos autores podemos definir la impulsividad como una conducta en la que prevalece un componente motórico de inmediatez, un comportamiento que también está asociado a factores cognitivos o en el que predominan aspectos emocionales-temperamentales.

Así, Moeller et al, mencionados por Gracia Marcoㄹ, definen la impulsividad como "una predisposición a reacciones rápidas no planificadas a estímulos externos o internos, sin consideración de las consecuencias negativas de esas reacciones para el individuo impulsivo o para los demás." En este mismo sentido se expresan Rodríguez Martínez y Ros Montalban², que encuentran en la impulsividad bajo umbral en el paso a la acción, enorme dificultad para valorar las repercusiones y escarmentar pese a las repetidas consecuencias de dicho comportamiento o para aprender o retrasarlo o evitarlo. Rubio Valladolid et $a l^{3}$ la definen como conductas pobremente planificadas, prematuramente realizadas, que representan cierto riesgo o son inapropiadas para la situación y a menudo conllevan consecuencias indeseadas.

Arranz, García y $\operatorname{Ros}^{4}$ mencionan a Evenden quien propuso que la conducta impulsiva presenta tres componentes derivados de las diferentes etapas del proceso de respuesta: preparación, ejecución y evaluación de los resultados. La impulsividad, para este autor, es una conducta que se manifiesta como resultado de la toma de decisión prematura, antes de disponer de toda la información necesaria (fase de preparación), también de la dificultad para realizar las acciones de forma apropiada (fase de ejecución) $y$, finalmente, de la incorrecta valoración de las consecuencias a largo plazo de las conductas que se realizan (fase de evaluación de las consecuencias).

Barrat, citado por Sqillace, Picón y Schmith ${ }^{5}$, distingue en la impulsividad tres factores: 1 . Impulsividad motora, que implica actuar sin pensar, dejándose llevar por las emociones del momento. 2. Impulsividad atencional, que supone un bajo control sobre las intrusiones de pensamientos y dificultades para la atención sostenida y 3 . Impulsividad por imprevisión, que se caracteriza por la tendencia a no planificar, mostrando un mayor interés por el presente que por el futuro. Patton, según refiere Gracia Marcoㄹ, encuentra tres partes en la impulsividad: a) actuar según el momento (activación motora), b) no centrarse en la tarea entre manos (atención) y c) no planificar ni pensar cuidadosamente.

Para otros autores el carácter de inmediatez de la respuesta no es el elemento esencial de la impulsividad. Así, Szerman ${ }^{6}$ cita a Newman y Wallace, quienes opinan que las conductas impulsivas de los sujetos antisociales reflejan la dificultad en el cambio automático de atención, que interfiere con su capacidad de asimilar información relevante mientras están ocupados en organizar una conducta dirigida a un objetivo.

Incluso algunos como Freeman, mencionado por Peris $^{7}$, encuentran un carácter deliberativo en la impulsividad. El autor cree que todos los Ilamados trastornos puros del control de los impulsos deberían considerarse, más que conductas caracterizadas por un inicio repentino, como el resultado de una extensa deliberación y larga lucha del individuo, incluso si la conducta aparece de repente para observadores externos. A estos trastornos los denomina "compulsivos". Ibáñez y Saiz ${ }^{8}$, refiriéndose a los trastornos del control de los impulsos, escriben que la realización del acto (impulsivo) en sí puede ser premeditada y planificada o no serlo.

Otros autores, desde un enfoque personológico, relacionan la impulsividad con determinados rasgos asociados a las emociones.

Cloninger reseña que en la conducta impulsiva intervienen cuatro rasgos heredables temperamentales: a) alta búsqueda de novedades, b) baja evitación de lesiones, c) baja persistencia y d) baja dependencia de la recompensa ${ }^{6}$. El autor ha postulado dos variables de la personalidad Ilamadas "Evitación del Daño" y "Búsqueda de Novedad". Las personas evitadoras del daño tienen tendencia a responder con inhibición conductual ante el castigo o frustración. Dentro del rasgo "búsqueda de novedades", distingue dos facetas, la "Exploración" que abarca a personas que muestran conductas de aproximación ante estímulos novedosos, se aburren fácilmente en situaciones rutinarias y tienden a desarrollar conductas adictivas y la "Impulsividad", en la que los sujetos son propensos a conductas impulsivas (Squillace, Picón y Schmidt) ${ }^{5}$.

Eysenck, como recuerda Gracia Marco ${ }^{1}$, incluye el rasgo "búsqueda de riesgo" en el componente 
"impulsividad" del factor "extroversión". Squillace y Picón y Schmidt) ${ }^{5}$ deducen que en la teoría de EysencK se podrían distinguir dos grandes impulsividades: la "impulsividad en sentido estricto" y la "búsqueda de aventuras". Zuckerman definió el rasgo "búsqueda de sensaciones" como la búsqueda de nuevas experiencias, excitación, desinhibición y susceptibilidad al aburrimiento ${ }^{1}$. Para Hollander ${ }^{9}$, en esta misma línea, la impulsividad se caracteriza por impaciencia, búsqueda de riesgo, preferencia por pequeñas recompensas inmediatas, búsqueda del placer y agresividad y disminución de la capacidad de análisis de las consecuencias.

\section{Los componentes de la impulsividad como bases biopsicológicas de la imputabilidad}

Un repaso bibliográfico del concepto de impulsividad nos ha permitido diferenciar los distintos elementos que intervienen en esta conducta: factores motóricos, cognitivos y temperamentales-emocionales. La influencia relativa que cada uno de ellos ejerce sobre la conducta impulsiva nos sugiere que ésta puede aparecer de forma inmediata o presentarse diferidamente. Esto nos permite distinguir entre las que podemos llamar "conductas impulsivas inmediatas" y "conductas impulsivas diferidas". Veremos a continuación como la diferente implicación de los componentes de estas conductas suponen repercusiones biopsicológicas sobre la imputabilidad de los sujetos que las llevan a cabo.

La jurisprudencia española tradicionalmente ha exigido complementar los criterios cualitativo, cuantitativo, cronológico y de causalidad para admitir una anomalía o alteración psíquica como causa eximente de responsabilidad penal.

Para satisfacer el criterio cualitativo (alteraciones cognitivas y/o volitivas) debemos hacer una interpretación holística de la impulsividad, estudiando no sólo la interacción de sus componentes motórico, cognitivo y temperamental-emocional, sino también analizándola como rasgo dentro de un constructo de personalidad determinado, como síntoma, en su caso, de algunas enfermedades mentales, como categoría diagnóstica diferenciada (Trastornos de control de los impulsos puros) o, finalmente, asociada a eventual consumo de sustancias psicoactivas.

Al estudiar el criterio cuantitativo no olvidemos la característica dimensional de la impulsividad, pues ésta es una conducta humana natural y necesaria ${ }^{9}$ que puede ser de utilidad en determinadas circunstancias como mecanismo adaptativo. Sólo a las desviaciones cuantitativas de esta conducta que denotasen, al decir de Cabrera y Fuertes, "la incapacidad de la razón para controlar las demandas de la afectividad o de los instintos"10 y que consecuentemente resultasen inadaptadas (perjudiciales para uno mismo o los demás) podrían ser consideradas como patológicas.

Los criterios cronológico y de causalidad exigen la concurrencia en el tiempo de la conducta impulsiva y el hecho ilícito y que éste sea consecuencia de aquélla. La interrelación de los componentes motórico, cognitivo y afectivo de la conducta humana con estímulos concretos pueden ayudar a identificar los actos punibles como respuestas comportamentales impulsivas a esos estímulos.

\section{Conductas impulsivas inmediatas}

En ellas tiene mayor importancia el componente motórico. Son irreflexivas, suponen una respuesta inmediata a estímulos, no tienen una motivación racional clara $^{11}$, resultan imposibles de inhibir y se manifiestan en "acting-out". Prevalece la recompensa inmediata y están dotadas de fuerte carga afectiva, fundamentalmente la ira (Sanz, Magán y García Vera) ${ }^{12}$.

Se corresponden con la "Impulsividad motora de Barrat" (actuar sin pensar, dejándose llevar por la emoción del momento), con la "activación motora" de Patton (actuar según el momento), con la impulsividad en sentido estricto de Eysenck, con la faceta "Impulsividad" del "rasgo de búsqueda de novedades" de Cloninger y con el factor "desinhibición" de la dimensión "búsqueda de sensaciones" de Zuckerman.

Este tipo de impulsividad es muy frecuente en las personas con trastorno límite en las que existe "predisposición a los arrebatos de ira y violencia" (CIE $X)^{13}$. Aparece en algunos comportamientos de los antisociales, que presentan "muy baja tolerancia a la frustración o bajo umbral para descargas de agresividad, dando incluso lugar a comportamiento violento" $(\mathrm{CIE} \mathrm{X})^{13}$ y es la característica esencial del trastorno explosivo intermitente donde aparecen episodios aislados de dificultad para controlar los impulsos agresivos, que resultan desproporcionados con respecto a la provocación o a la intensidad del estresante psicosocial precipitante (DSM IV TR) ${ }^{14}$.

Desde un punto de vista neuropsicológico se ha demostrado en las personas impulsivas una disfunción en los circuitos prefrontales (zona orbitofrontal, ventromedial y córtex cingulado anterior) junto con una alteración de varias estructuras 
subcorticales (amígdala, hipocampo e hipotálamo), sus conexiones y en el equilibrio relativo de la actividad de estas regiones ${ }^{15}$.

Goyer, citado por Raine ${ }^{16}$, demostró mediante tomografía de emisión de positrones, que los actos impulsivos crecían a medida que descendía la cantidad de glucosa en la corteza frontal en 17 pacientes con trastornos de la personalidad. Este mismo autor estudió a asesinos depredadores y asesinos afectivos (impulsivos) encontrando en ambos grupos mayores tasas de actividad en la amígdala, hipocampo e hipotálamo y tasas bajas de actividad en la córtex prefrontal de los asesinos afectivos. Anormalidades en el metabolismo del córtex ventromedial y orbitofrontal se han hallado, además de en sujetos antisociales, en el trastorno límite de la personalidad y en trastorno explosivo intermitente ${ }^{17}$.

La demostración de lesiones estructurales prefrontales y subcorticales en sujetos impulsivos adquieren especial relevancia a la hora de evaluar las alteraciones cognitiva o volitiva en ellos. Estas estructuras cerebrales están implicadas en la denominada "función ejecutiva" 15 , que se define como el proceso por el cual se logra planificar, anticipar, inhibir respuestas, desarrollar estrategias, juicios y razonamientos de acuerdo a las exigencias y demandas sociales y personales.

De esta forma, el daño de las regiones orbitofrontales y ventromediales se relaciona con déficit en el proceso de toma de decisiones, falta de regulación conductual en respuesta a la aparición de estímulos ambientales, alteración en el razonamiento moral, dificultad para acceder a ciertos conocimientos sociales como interpretar las expresiones emocionales no verbales e inadecuado procesamiento de la información emocional. Las anormalidades en la actividad del hipotálamo y amígdala se asocian a evaluación imprecisa de los estímulos del ambiente, que son percibidos como amenazantes, facilitando la aparición de ira ${ }^{18}$.

Por tanto, encontramos en los sujetos con impulsividad inmediata alteraciones de carácter cognitivo, emotivo y conductual que afectan a la función ejecutiva y modifican, si quiera parcialmente, las bases biopsicológicas de la imputabilidad, y así es reconocido por autores como Cabrera y Fuertes ${ }^{10}$.

Algunos comportamientos ilegales por su aparición repentina e irreflexiva, irresistible, desproporcionada o íntimamente ligada a estímulos concretos, pueden ser atribuibles cronológica y causalmente a la impulsividad del sujeto que los comete, si bien, hemos de reconocer con Carrasco ${ }^{19}$ la dificultad que entraña apreciar la intensidad de la irresistibilidad de estas conductas impulsivas.

\section{Conductas impulsivas diferidas}

Llamamos así a las conductas deliberadas que surgen tras una decisión impremeditada y tiene como finalidad llevar a cabo dicha decisión.

En estas conductas, más que el componente motórico inmediato, adquieren relevancia elementos cognitivos y temperamentales-emocionales. Se aprecia un fracaso en resistir el impulso de llevar a cabo una conducta; en palabras de González, citado por Carrasco ${ }^{11}$, hay pérdida de control o pérdida de habilidad para dejar de hacer una conducta. Existe por tanto y en primer lugar, una decisión precipitada, pero posteriormente se desarrollan actos premeditados que pueden ir asociados a una activación emocional o búsqueda de excitación, que culminan con una experiencia de placer.

En algunos aspectos podrían ser equivalentes a la "Impulsividad atencional de Barrat", por la aparición de pensamientos intrusivos y en otros a la "impulsividad por imprevisión" del mismo autor (tendencia a no planificar, mayor interés por el presente que por el futuro). En terminología de Evenden, se deben a una alteración en la fase de evaluación de los resultados, apareciendo una incorrecta valoración de las consecuencias a largo plazo de las conductas que se realizan. Para este autor, la mala evaluación de los resultados es propia de las conductas adictivas.

Este tipo de conducta impulsiva es característica, en nuestra opinión, del juego patológico y la piromanía (incluidos en DSM IV TR en trastornos puros del control de impulsos) y de algunos sujetos antisociales.

En el juego patológico como lo define DSM IV TR, existe un fracaso repetido de los esfuerzos para controlar, interrumpir o detener el juego. El ludópata planifica la próxima aventura de juego o piensa en la forma de conseguir dinero para seguir jugando. La piromanía consiste en la presencia de múltiples episodios de provocación de incendios de forma deliberada e intencionada. Los pirómanos suelen hacer considerables preparativos para provocar un incendio ${ }^{20}$.

Los elementos cognitivos de estas conductas impulsivas juegan un papel fundamental tanto en el inicio como en el mantenimiento de las mismas, como aseguran Robert y Botella en relación al juego patológico ${ }^{21}$. En este trastorno, por ejemplo, se observan distintas distorsiones cognitivas como el pensamiento mágico ${ }^{4}$, la "ilusión de control", la percepción de autoeficacia en relación con la probabilidad de ganancias y la evaluación sesgada de los resultados del juego (Echeburua, 1992) ${ }^{22}$. Para Evenden, además, la incorrecta valoración de las consecuencias a largo plazo es propia de las conductas 
adictivas ${ }^{4}$. En este sentido podríamos afirmar que los jugadores patológicos sufren "impulsividad no planificadora", es decir, una incapacidad para planificar el futuro utilizando la información disponible $y$, por tanto, para pensar, evaluar y prever las consecuencias de los actos, según Barrat. Pero en estas conductas existen también pensamientos intrusivos difíciles de controlar, como se postula en la Impulsividad atencional del mismo autor.

Factores temperamentales y emocionales como el "arousal" y "la búsqueda de sensaciones" de Zuckerman se han señalado como variables significativas en la adquisición del juego patológico ${ }^{22}$. Ello es así porque, para el autor, los buscadores de estimulación perciben los riesgos como menores y la excitación anticipada como más positiva. Covertry y Brown ${ }^{23}$ sugieren que la búsqueda de sensaciones es una variable muy importante tanto en la elección del juego como en la forma que éste se desarrolla. No obstante, como apuntan Fernández y Echeburua ${ }^{24}$, la relación entre la dimensión "búsqueda de sensaciones" y la ludopatía no es tan clara y unívoca. Parece que esta dimensión, más que una característica común a todos los jugadores, solo puntúa alta en aquellos para los que el juego es especialmente reforzante debido a las intensas emociones inherentes al mismo ${ }^{21}$.

Desde la perspectiva médico-legal, consideramos que estos elementos cognitivos y temperamentales pueden influir en las bases biopsicológicas de la imputabilidad del paciente con estos trastornos. En efecto, por un lado, el pensamiento mágico, las distorsiones cognitivas y la incapacidad para prever el futuro y evaluar las consecuencias de los actos y, por el otro, la dimensión "búsqueda de sensaciones", es decir, "búsqueda de emociones, de situaciones excitantes sobre una base de intranquilidad, disforia e inquietud, desinhibición y evitación de la rutina", como describe Zuckerman ${ }^{6}$, junto con la pérdida del control o la pérdida de habilidad para dejar de hacer esa conducta ${ }^{11}$, pueden influir en las capacidades cognitiva y volitiva y disminuir la responsabilidad criminal cuando los delitos cometidos se relacionan teleológicamente con la conducta impulsiva.

Carrasco ${ }^{11}$ encuentra igualmente una base patológica en la ludomanía como para plantearse cierta modificación de los fundamentos biopsicológicos de la imputabilidad, al menos como atenuante o incluso como eximente incompleta en casos graves. En este mismo sentido se expresan Cabrea y Fuertes ${ }^{10}$ para quienes existe en este trastorno una perturbación severa del control volitivo, aunque puntualizan la necesidad de analizar cada caso concreto.

Algunos de los trastornos puros del control de los impulsos, por nosotros considerados conductas impulsivas diferidas, como el juego patológico, son conceptualizados como conductas adictivas o adicciones no tóxicas (Blaszczynski, Buhrich y McConaghy; Custer y Milt; Dickerson; Echeburua, García, Díaz y Aranda; Rodríguez Marcos; Sainz y López-lbor, recogidos por Robert y Botella) ${ }^{21}$. Son comportamientos que producen adicción, y comparten algunos factores etiológicos y síntomas (tolerancia o dependencia) con las adicciones a sustancias (Lesieur y Heineman, Volberg y Steadman, mencionados por Echeburua) ${ }^{22}$. Cabría plantearse, por tanto, si los elementos psicopatológicos que intervienen en la modificación de responsabilidad penal de las adicciones tóxicas son también aplicables a las conductas adictivas no tóxicas.

Especial interés médico-legal tienen las más controvertidas conductas impulsivas diferidas en sujetos antisociales.

En efecto, observamos que ciertos comportamientos de las personas antisociales podrían encajar en el concepto propuesto por nosotros de "conductas impulsivas diferidas", en las que tras una decisión precipitada e irreprimible, adquieren preeminencia los componentes cognitivos y temperamentales.

Entre los componentes cognitivos, el criterio A3 de trastorno antisocial de la personalidad de DSM IV $\mathrm{TR}^{25}$ describe en estos sujetos "impulsividad o incapacidad para planificar el futuro" y el mismo texto explica que "las decisiones se toman sin pensar, sin prevenir nada y sin tener en cuenta las consecuencias para uno mismo o para los demás, lo que puede originar cambios repentinos de lugar de trabajo, de residencia o de amistades".

Pensamos que estas conductas pueden ser explicadas por la teoría ya mencionada de "la deficiente respuesta a la modulación" de Newman y Wallace. Según los autores, las conductas impulsivas de los sujetos antisociales reflejan la dificultad en el cambio automático de atención, que le incapacitan para asimilar información relevante mientras están ocupados en organizar una conducta dirigida a ese objetivo. Para nosotros, este modelo podría explicar algunos comportamientos impulsivos deliberados que aparecen en estos individuos (cambios repentinos de residencia, trabajo, etc. y algunas conductas depredadoras). Por ejemplo, el sujeto que decide repentinamente cambiar de vivienda sin tener en cuenta las consecuencias futuras de esta decisión (repercusiones en su trabajo, en la familia, etc.). La decisión primera (cambiar de vivienda) es irreflexiva, pero a partir de ese momento el sujeto concatena distintas conductas deliberadas encaminadas a buscar una nueva vivienda, a obtener financiación de la misma, a desprenderse de la antigua, etc.

También es observable en estos comportamientos, como en las conductas adictivas, una incapacidad 
para planificar el futuro ("impulsividad no planificadora" de Barrat) o una falla en la evaluación de las consecuencias de los actos, como describió Evenden.

Desde la perspectiva temperamental el sujeto antisocial se caracteriza por una dimensión alta de "búsqueda de sensaciones". Millon ${ }^{26}$ describe a estas personas como incapaces de soportar el tedio y la rutina con facilidad para aburrirse e inquietarse. Presentan marcada propensión a buscar desafíos y riesgos, actuando como si fueran inmunes al peligro. Tienen -continúa el autor- una pasión por la vida que les impulsa a perseguir la excitación y los placeres... un impulso para explorar lo prohibido... Algunos de ellos "son buscadores de peligros, fácilmente seducidos por las oportunidades de probarse a sí mismos". Autores como Pérez, Ortet, Pla, Simó, Rutter, Guiller o Hagell, mencionados por López y López ${ }^{27}$ también han relacionado la variable "búsqueda de sensaciones" con la conducta antisocial. Para Horvarth y Zuckerman ${ }^{28}$ la alta búsqueda de sensaciones es un rasgo mayor de los psicópatas, que parecen casi inconscientes del riesgo de sus actividades criminales, en comparación a los criminales no psicopáticos.

Neuropsicológicamente, el estudio ya referido de Goyer encontró altas tasas de actividad en la amígdala, hipocampo e hipotálamo tanto en asesinos depredadores (controlados, planificadores) como en asesinos afectivos (impulsivos) y disminución de actividad en la córtex prefrontal de los asesinos afectivos. En esta misma línea, Raine et a/29 informan del relativamente normal funcionamiento de la corteza prefrontal en agresores instrumentales (depredadores). Sin embargo, Hare ${ }^{30}$ atribuye el comportamiento desinhibido de los psicópatas, incluyendo su facilidad para la violencia depredadora, a una disfunción en la corteza frontal ventromedial (integración cognitiva-afectiva) y en la corteza frontal dorsolateral (inhibición de la respuesta) y/o con una comunicación ineficaz con otras áreas del cerebro. Para Zuckerman 6 el rasgo "búsqueda de sensaciones" es consecuencia de un estado deficitario de activación cortical que lleva al sujeto a la búsqueda de situaciones excitantes, sobre una base de intranquilidad, disforia, inquietud y evitación de la rutina. Según Dahl y Hellkige, citados por Raine ${ }^{14}$, la corteza prefrontal está envuelta en la regulación de la activación y se piensa que precisamente son deficiencias en la regulación de la activación del sistema autónomo central las que llevan a los antisociales a buscar estimulantes que compensen esa baja activación.

Nuestra hipótesis es que comportamientos aparentemente deliberados de sujetos antisociales tienen características comunes con conductas impulsivas y comparten con ellas similares fundamentos cognitivos, emocionales y neuropsicológicos. Por tanto, ¿están afectos igualmente de alguna merma en las capacidades cognitiva y volitivas?

Desde una perspectiva cognitiva la teoría de Newman y Wallace de "la deficiente respuesta a la modulación" que implica en los sujetos antisociales una dificultad para el cambio de atención que le incapacita para asimilar información relevante mientras está ocupado en organizar una conducta dirigida a ese objetivo, podría tener una cierta relevancia en la capacidad cognitiva con respecto a esa conducta. Igualmente, la incapacidad de análisis y crítica de las consecuencias de los actos o las dificultades para planificar el futuro pueden tener repercusiones en la capacidad cognitiva.

Por otro lado, encontramos anomalías cognitivas en la incapacidad que tienen los sujetos antisociales, debido a lesiones amigdalares e hipocámpicas ${ }^{31}$ para reconocer y evaluar correctamente ciertos estímulos afectivos socialmente significativos y de la que se derivan comportamientos socialmente inadecuados. Pero también -creemos- podría existir afectación en la voluntariedad del psicópata , pues, siguiendo a Hare ${ }^{30}$, la conducta de este sujeto no se ve modificada (a causa de disfunción de la corteza frontal ventromedial y dorsolateral) por las emociones que inhiben el comportamiento de una persona normal, permitiéndole cometer actos depredadores y violentos sin ningún escrúpulo.

Si como propone Zuckerman, mencionado por Alcázar Córcoles ${ }^{32}$, los individuos con alta dimensión "búsqueda de sensaciones" tienen fuertes "motores" de aproximación a los estímulos nuevos y gratificantes y débiles "frenos" conductuales (por ejemplo, se inhiben menos ante las señales de castigo y, por tanto, son menos disuadidos por las consecuencias negativas de la delincuencia) ino existiría en estos sujetos una cierta incapacidad de controlar sus actos voluntarios?

Finalmente, el concepto de conducta adictiva no tóxica también está siendo aplicado a determinados comportamientos de personas antisociales. Hodge, mencionado por Garrido ${ }^{33}$, mantiene la tesis de que un proceso adictivo juega un papel de primer orden en la conducta criminal, incluyendo el asesinato en serie y la delincuencia sexual. "El elemento común afirma el autor- es que el delito es cometido para alcanzar un estado experiencial antes que por los motivos más usuales de ganancia financiera o la venganza". Un trabajo realizado por Greswell en $1991^{33}$ con asesinos múltiples y sexuales confirmó que esas personas mostraban conductas y fantasías características del proceso adictivo. Skrapec ${ }^{34}$ en un estudio con asesinos en serie describe sensaciones que, en nuestra opinión, podrían ser elementos propios de las conductas adictivas. Así el autor relata que "sentían un éxtasis eufórico o una ira 
violenta que al exteriorizar (matando) les producía gran placer. Después de esto le sobrevenía un estado de calma y una sensación de alivio. Pero, según dijeron todos, no lograban conservar estas sensaciones después del asesinato y cada vez se encontraban más intranquilos. Para hacer frente a este estado de intranquilidad se refugiaban en el alcohol o en sus fantasías; pero, al final, el único modo de encontrar la tranquilidad (temporalmente) era volver a matar".

Entonces podríamos preguntarnos si a estas conductas depredadoras deliberadas y reiteradas 0 a otras similares de sujetos antisociales se les pueden aplicar los mismos condicionantes sobre la responsabilidad penal que a las conductas adictivas.

Aunque finalmente hallásemos en determinados comportamientos ilícitos de los antisociales elementos cognitivos o temperamentales que nos permitiesen hablar de impulsividad diferida y por tanto con influencia en las capacidades cognitiva y volitiva, hemos de reconocer las dificultades que entraña cuantificar dicha influencia y establecer teleológicamente nexos cronológico o de causalidad.

\section{Conclusiones}

En la impulsividad intervienen distintos componentes, el motórico, el cognitivo y el temperamental-emocional. Cada uno de ellos adquiere una importancia relativa en las que hemos denominado "conductas impulsivas inmediatas" y conductas impulsivas diferidas".

El conocimiento de los distintos factores de estos comportamientos impulsivos y su interacción nos ayudan a establecer los elementos de influencia en las capacidades cognitiva y volitiva y así facilitar a los tribunales la valoración de la imputabilidad de estas conductas.

En efecto, las conductas impulsivas inmediatas se caracterizan por un importante componente motórico y fuerte carga afectiva, con presentación en "acting-out". Tienen como correlatos neuroanatómicos disminución de la actividad de la corteza prefrontal y ventro- medial y aumento de la actividad en amígdala, hipocampo e hipotálamo. Ello representa alteraciones en la "función ejecutiva" que merman, en alguna medida, las capacidades cognitiva y volitiva. Los criterios cronológico y de causalidad no son difíciles de cumplimentar, aunque pueden presentarse dificultades para cuantificar la intensidad de estas conductas.

En las conductas impulsivas diferidas existe una primera decisión impremeditada seguida de comportamientos elaborados y complejos que tiene como finalidad llevar a cabo la decisión inicial. Intervienen sobre todo distorsiones cognitivas y factores temperamentales-emocionales y, aunque aún no están lo suficientemente estudiadas, se han relacionado con alteraciones en la corteza frontal y en regiones subcorticales. Encontramos que el juego patológico, la piromanía o algunos comportamientos premeditados y depredadores de los antisociales responden a este patrón de impulsividad. Existe una cierta unanimidad en cuanto a considerar el juego patológico como un trastorno del control de los impulsos y a sus implicaciones médico-legales. Por el contrario, las conductas depredadoras de los psicópatas no son consideradas por la mayoría de los autores como actos impulsivos, si bien algunos hallan similitudes con las adicciones no tóxicas. Por nuestra parte, hemos creído encontrar elementos en estos comportamientos que nos permiten clasificarlos como conductas impulsivas diferidas y que, por tanto, podrían interferir en las capacidades volitiva y cognitiva del sujeto que las ejecuta. Hay que reconocer, no obstante, las dificultades que existen para satisfacer los requisitos legales de afectación de la imputabilidad.

Las investigaciones con neuroimagen están evidenciando alteraciones funcionales de las áreas cerebrales implicadas en la impulsividad; tal vez una profundización en el estudio neuroanatómico y biopsicológico de estas regiones ayuden a dilucidar en el futuro algunas de las cuestiones médico-legales aquí planteadas.

En todo caso, el diagnóstico de la conducta impulsiva y su implicación forense debe realizarse en el contexto más amplio de constructo de personalidad, de enfermedad mental o de consumo de sustancias.

\section{Bibliografía}

1. Gracia Marco R. Nosología de la Impulsividad. En: Ros Montalbán S, Peris Díaz MD, Gracia Marco R. Impulsividad. $1^{a}$ ed. Barcelona: Ars Médica, 2004. p. 17-22.

2. Rodríguez Martínez A, Ros Montalbán S. Los trastornos puros. En: Ros Montalbán S, Peris Díaz MD
Gracia Marco R. Impulsividad. $1^{\text {a }}$ ed. Barcelona: Ars Médica, 2004. p. 81-99.

3. Rubi G, Rodríguez R, Ponce G, Jiménez-Arriero MA, San Molina L, Palomo T. Impulsividad y abuso de sustancias. En: Ros Montalbán S, Peris Díaz MD, Gracia Marco R. Impulsividad. $1^{\text {a }}$ ed. Barcelona: Ars Médica, 2004. p.119-40. 
4. Arranz FJ, García S, Ros S. Modelos experimentales de investigación de la impulsividad. En: Ros Montalbán S, Peris Díaz MD, Gracia Marco R. Impulsividad. $1^{\text {a }}$ ed. Barcelona: Ars Médica, 2004. p. 23-39.

5. Squillace M, Picón J, Schmidt V. El concepto de impulsividad y su ubicación en las teorías psicobiológicas de la personalidad. Rev Neuropsicológica Latinoamericana. 2011;3(1):8-18.

6. Szerman N. Impulsividad y trastornos de la personalidad. En: Ros Montalbán S, Peris Díaz MD, Gracia Marco R. Impulsividad. $1^{\text {a }}$ ed. Barcelona: Ars Médica, 2004. p. 101-17.

7. Peris MD. Impulsividad versus compulsividad. En: Ros Montalbán S, Peris Díaz MD, Gracia Marco R. Impulsividad. $1^{\text {a }}$ ed. Barcelona: Ars Médica, 2004. p. 211-28.

8. Ibañez A, Saiz J. Trastornos de los hábitos y del control de los impulsos. En: Barcia D. Tratado de psiquiatría. Madrid: Ed. Aran ediciones, 2000. p. 579-602.

9. García G. Neuroanatomía de la impulsividad. En: Ros Montalbán S, Peris Díaz MD, Gracia Marco R. Impulsividad. $1^{a}$ ed. Barcelona: Ars Médica, 2004. p. 43-51.

10. Cabrera J, Fuertes JC. Psiquiatría y Derecho. Madrid: Ed Cauce, 1997. p. 313-21.

11. Carrasco JJ, Maza JM. Tratado de Psiquiatría Legal y Forense. $4^{\circ}$ ed. Madrid: Ed La Ley, 2010. p. 1599-629.

12. Sanz J, Magán I, García MP. Personalidad y el síndrome AHÍ (agresión -hostilidad-ira): Relación de los cinco grandes con ira y hostilidad. Psicopatología Clínica, Legal y Forense 2006;6:153-70.

13. OMS: CIE X Trastornos mentales y del comportamiento. Madrid: Ed Meditor, 1992. p. 245-76.

14. DSM IV TR Manual diagnóstico y estadístico de los trastornos mentales. Barcelobna: Ed Masson, 2002. p. 741-57.

15. Alcázar-Córcoles MA, Verdejo-García A, Bouso-Sainz JC, Bezos-Saldaña L. Neuropsicología de la agresión impulsiva. Rev Neurol 2010;50:291-9.

16. Raine A. Psicopatía, violencia y neuroimagen. En: Raine A, Sanmartín J. Violencia y psicopatía. BarceIona: Ed Ariel, 2000. p. 59-88.

17. Barez NB, Fernández S. Repercusiones forenses del daño en el cortex prefrontal y ventromedial: Relevancia de la toma de decisiones. Psicopatología Clínica, Legal y Forense 2007; 7:127-45.

18. Rodríguez MJ, Fernández S. Disfunción neuropsicológica en maltratadores. Psicopatología Clínica, Legal y Forense 2006;6:83-101.

19. Carrasco JJ, Maza JM. Tratado de Psiquiatría Legal y Forense. $4^{\circ}$ ed. Madrid: Ed La Ley, 2010. p. 282-351.
20. DSM IV TR Manual diagnóstico y estadístico de los trastornos mentales. Ed Masson. Barcelona, 2002. p. 741-58.

21. Robert C, Botella C. Trastornos del control de los impulsos: El juego patológico. En: Beloch A, Sandín B, Ramos F. Manual de Psicopatología. Barcelona: Ed. Mc Grawhill, 2008. p. 424-48.

22. Echeburua E. Psicopatología, variables de personalidad y vulnerabilidad psicológica al juego patológico. Psicothema, 1992;4(1):7-20.

23. Coventry KR, Brown IF. Sensation Seeking and gambling addictions. Addict, 1993;88:541-54.

24. Fernández J, Echeburua E. Juego Patológico y trastornos de personalidad: un estudio piloto con el MCMIII. Psicothema 2006;18(3):453-8.

25. DSM IV TR Manual diagnóstico y estadístico de los trastornos mentales. Barcelona: Ed Masson, 2002. p. $765-816$

26. Millon T, Roger D. Trastornos de la personalidad. Mas allá del DSM IV. Barcelona: Ed Masson, 2000. p. 447-92.

27. López Soler C, López López JR. Rasgos de la personalidad y conducta antisocial y delictiva. Psicopatología Clínica, Legal y Forense 2003;3(2):5-19.

28. Horvarth P, Zuckerman M. Búsqueda de sensaciones, valoración y conducta de riesgo. Rev de Toxicomanías 1996;9:26-38.

29. Raine A, Melroy JR, Birhle S, Stoddard J, La Casse L, Buchsbaum MS. Reduced prefrontal and increased subcortical brain functioning assessed using possitron emisión tomography in predatory and affective murderes. Behav Sci Law. 1998;16(3):319-32.

30. Hare R. La naturaleza del psicópata: Algunas observaciones para entender la violencia depredadora humana. En: Raine A, Sanmartín, J. Violencia y psicopatía. Barcelona: Ed. Ariel, 2000. p. 15-57.

31. Dodge KA, Prica Jm, Bachorowski JA, Newman JP. Hostile atributional bases in severely aggressive adolescents. J Abnorm Psychol. 1990;99:385-92.

32. Alcázar Córcoles MA. Patrones de Conducta y personalidad Antisocial en Adolescentes. Estudio transcultural: El Salvador, México y España (tesis doctoral). Madrid: Universidad Autónoma de Madrid, 2007.

33. Garrido V. El psicópata como entidad psicológica y cultural. En: Echeburua, E. Personalidades violentas. Madrid: Ed. Pirámide, 2000. p. 67-80.

34. Skrapec C: Los motivos del asesino en serie. En: Raine A, Sanmartín, J. Violencia y psicopatía. Barcelona: Ed Ariel, 2000. p. 155-80. 